\title{
LA DOMÓTICA COMO MEDIO PARA LA VIDA INDEPENDIENTE DE DISCAPACITADOS Y PERSONAS DE LA TERCERA EDAD
}

\author{
(INTELLIGENT HOUSES FOR ELDERS AND HANDICAPPED TO HAVE AN INDEPENDANT LIFE)
}

Alfonso Recuero, Dr. Ingeniero de Caminos

Instituto de Ciencias de la Construcción Eduardo Torroja (CSIC)

ESPAÑA

Fecha de recepción: 21-XII-98

$106-10$

\section{RESUMEN}

El colectivo de discapacitados y personas de la tercera edad puede cifrarse en 80 millones en la Unión Europea y es de esperar que aumente en los próximos años. El mantener y mejorar su calidad de vida incluye aspectos tales como facilitar su vida independiente, promover una mayor integración social, aumentar sus posibilidades de educación, formación y empleo, y mejorar la disponibilidad y calidad de su cuidado y asistencia.

Debe producirse una evolución socio-cultural en la forma de atender las necesidades de este colectivo, pasando de la situación paternalista, autoritaria y centralizada existente a otra más dirigida por el propio colectivo. Es necesario desarrollar criterios sobre la calidad de vida y calidad de los cuidados, criterios económicos, cómo pueden ser suministrados, etc.

Es necesario realizar investigaciones, entre otros, en aspectos médicos, sociales, económicos y tecnológicos. Desde el punto de vista de la Domótica serán estos últimos en los que se hará mayor hincapié, pero siempre insertos en $u$ orientados por los otros. Es importante reforzar el concepto de diseño para todos, haciendo que se tengan en cuenta las limitaciones impuestas por las discapacidades más habituales al diseñar los aparatos o servicios de uso general.

\section{SUMMARY}

The number of elders and handicapped is around 80 millions in the European Union, and this number supposedly will increase during the next few years. To have them live a better life includes having them independant, provide for them more social integration, increase their education, training and employment opportunities, and improve the availability and quality of health care and assistance.

A social and cultural evolution must take place in the ways care is provided to this group, going from the actual situation which is paternalistic, authoritarian and centralized, to another able to be controled by the own group. It is necessary to establish criteria on the life and health care quality, on the economy and how it has to be managed.

Among others, investigations must be conducted on medical, social, economic, and technological aspects. As for Intelligent Houses this aspect will be a priority within, or oriented by, the previous ones. It is important to reinforce the design concept for all of them, taking into account the most common disabilities when designing appliances or services of general use.

\section{Introducción}

La fracción de este colectivo respecto a la población total está aumentando. Por una parte se está reduciendo el número de niños y jóvenes, en tanto que crece la esperanza de vida. En la actualidad, el número de personas mayores de 60 años en la UE es del $20 \%$ y se espera que en el 2020 este porcentaje sea del $27 \%$, aumentando también el número de personas muy ancianas, con el $8,9 \%$ por encima de 75 años y, el 2,4\%, por encima de los 85 . Dada la mayor supervivencia de las mujeres, el colectivo de personas de la tercera edad será mayoritariamente femenino. 
A los 60 años la expectativa de vida es de unos 20 años, gran parte de ellos con una buena calidad de vida. Con cuidados adecuados, los años activos y con buena calidad de vida pueden aumentarse, pero, en cualquier caso, a medida que aumenta la edad, se producen una serie de cambios fisiológicos, acompañados habitualmente de pérdidas de funcionalidad, tales como pérdida de fuerza muscular, de visión, de capacidad auditiva, etc., que afectan a su capacidad de vida independiente. Entre la tercera y la cuarta parte de los mayores de 70 años necesitan asistencia en su vida diaria, mientras que el $30 \%$ de los mayores de 80 años padecen discapacidades severas. Todo esto hace que la edad avanzada y las discapacidades estén muy relacionadas.

Aproximadamente el $70 \%$ de los discapacitados tienen más de 60 años. Además de las discapacidades relacionadas con la edad, se está produciendo un aumento en el número de discapacitados debido al descenso de mortalidad por enfermedades graves, traumatismos, enfermedades pre $\mathrm{y}$ posnatales, $\mathrm{y}$ al aumento del número de accidentes $\mathrm{y}$ a la mejoría en la supervivencia de los mismos.

Dentro de este colectivo pueden distinguirse tres grupos que comparten necesidades comunes y tienen, a su vez, problemas específicos. Estos grupos son -por orden de importancia numérica- la mayor parte de personas de la tercera edad, que pueden tener o no alguna discapacidad, mayores con alguna discapacidad y discapacitados jóvenes. Entre sus demandas para poder desarrollar una vida activa e independiente pueden citarse: los cuidados de larga duración, continuos o intermitentes; oportunidades para mantener o mejorar las habilidades personales; autocuidados o cuidados a distancia; entornos domésticos

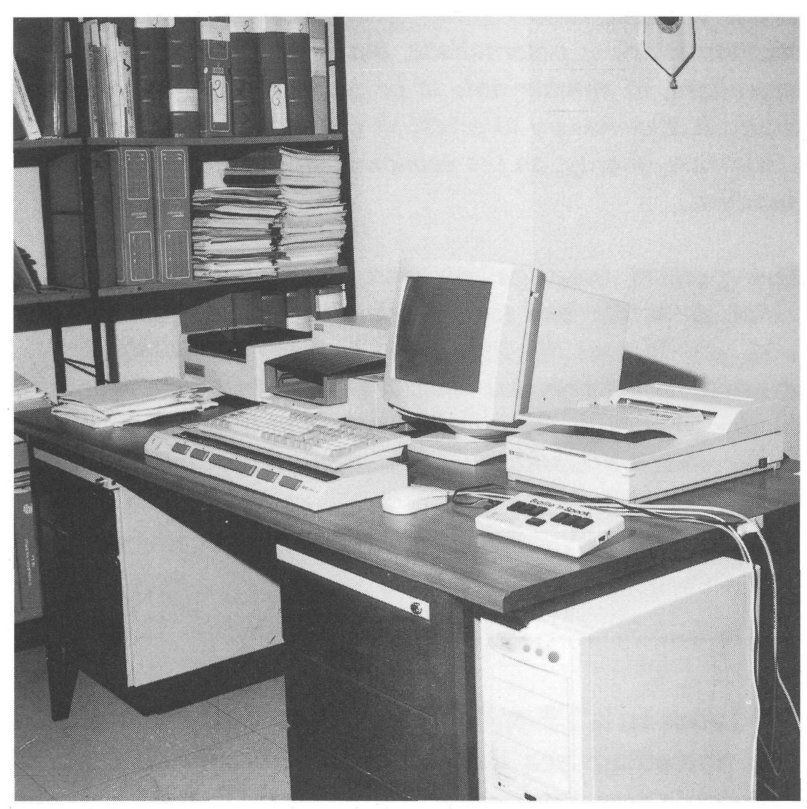

Adaptación de un equipo informático para su uso por un ciego, incluye sintetizador de luz "braille hablado" (a la derecha), linea "braille Eco 80", e impresora braille. acondicionados; productos de apoyo a sus deficiencias con tecnologías avanzadas; servicios que faciliten su vida independiente, etc. Evidentemente es necesario un enfoque multisectorial en el intento de satisfacer estas demandas, pero la Domótica tiene un papel importante en ello.

En este aspecto se ha producido un cambio importante por la evolución de la familia nuclear tradicional, aumentando las familias monoparentales, las personas que viven solas o compartiendo la vivienda con otras no relacionadas, que son situaciones con menor capacidad de asistencia, lo que hace crecer la importancia de la prestación de la misma por parte de organizaciones públicas o privadas.

Los avances tecnológicos juegan un papel central en estos aspectos, pero pueden ser contraproducentes si no se aplican adecuadamente. Por ejemplo, los audífonos pueden acoplarse magnéticamente con los teléfonos analógicos pero no con los digitales que los están sustituyendo, en tanto que la proliferación de los elementos gráficos en las aplicaciones y comunicaciones informáticas dificulta $o$ impide el acceso de los ciegos a las mismas.

La Informática y las comunicaciones unidas a avances en microelectrónica, optoelectrónica, desarrollo de nuevos materiales, ingeniería mecánica, con tecnologías altas, medias y bajas, participan en la creación de servicios y de dispositivos. El que las personas de la tercera edad y los discapacitados puedan hacer uso de ellos puede abordarse de dos formas: diseño universal, o para todos, o diseño especialmente adaptado o asistido.

En el primer caso, en el diseño de aparatos, programas o servicios de uso general se tienen en cuenta, desde un principio, las necesidades especiales de algunos usuarios, utilizando caracteres grandes y bien contrastados, marcas táctiles, descripciones adicionales a los gráficos, indicadores luminosos, configuraciones accesibles y manejables de los mandos, etc. Esto permite su utilización por una parte importante del colectivo sin que se incremente su precio. El caso de diseño asistido se reserva a la satisfacción de necesidades específicas de una discapacidad. En este caso los dispositivos suelen ser producidos por empresas pequeñas o medianas, de carácter nacional, lo que lleva a que sean productos caros, faltos de normalización, incompatibles entre sí y que no evolucionan con la misma rapidez que el resto. En lo posible, estos dispositivos deberían complementar a los primeros, de forma que pudieran ser utilizados junto a varios modelos o a versiones sucesivas.

\section{Sistemas domóticos de información}

Los sistemas domóticos de información han sido considerados hasta ahora como soportes pasivos, meras herramientas necesarias para el-funcionamiento de los dispositivos de ayuda a los discapacitados. En un futuro 
deberán también ser tenidos en cuenta como elementos activos, como medio de conexión con otras actividades exteriores que pueden ampliar sus perspectivas de vida.

Las funciones de estos sistemas son:

-El registro y gestión de la información: por ejemplo, de los consumos de los servicios, tales como agua gas electricidad; control físico y cuidado de plantas y animales domésticos; control ambiental de la casa, etc.

-La confirmación y recuperación de la información: monitorizando aquello que ocurre en otras habitaciones o en el exterior de la vivienda; inventario de contenidos en desvanes, trasteros $\mathrm{u}$ otro tipo de almacenamientos; localización de cosas de uso poco frecuente, etc.

-La presentación de la información: métodos, dispositivos, lugar y momento adecuados.

-La recepción de mensajes y reconocimiento de emergencias: instalación de alarmas, sensores, aparatos de información, etc.

-La emisión y recepción de información y conexiones: envío de información a personas específicas o inespecíficas; posibilidades y métodos de transmisión de información en viajes.

- En la educación y esparcimiento: el placer de disponer y utilizar la información; educación o cuidado de la casa asistidos por ordenador, etc.

Para cumplir con estas funciones, los sistemas domóticos de información para discapacitados o personas mayores deberían disponer de una interfaz con el usuario de manejo fácil ("a prueba de tontos"), con interacciones gráficas, etc., y de sistemas con control de fallos, con recuperación del sistema, suministro en cortes eléctricos o caídas de tensión, etc.

Deben satisfacerse también distintos tipos de requerimientos para poder integrar en la vivienda este tipo de sistemas. Unos son de tipo constructivo, tales como: instalar los cableados suficientes y dejar canalizaciones que permitan instalar nuevos cableados en el futuro; prever espacios adecuados para equipos grandes, tales como pantallas; situar los enchufes, paneles de control y conmutadores con criterio ergonómico, teniendo en cuenta las necesidades específicas; planear distribuciones y utilizar materiales y estructuras que no interfieran los rayos infrarrojos o las ondas eléctricas que se utilizan para el control remoto de aparatos; proteger los dispositivos microelectrónicos frente a la humedad, el calor, etc.; disponer de espacios o servicios comunes para atención o asistencia, tales como sistemas de recepción de correo y mensajes con atención personal, etc.

Otros son de tipo informativo o conceptual, tales como: facilitar explicación sobre los sistemas de información integrados en la vivienda y sobre sus posibilidades de expansión; proporcionar manuales de usuario para el manejo y el mantenimiento de los sistemas; indicar los métodos de comunicación con otros ocupantes del inmueble y formas de evacuación en casos de emergencia; presentar propuestas para modos de vida, seguridad cotidiana, etc.

Y otros se refieren a las prestaciones de los servicios de soporte a los distintos sistemas y sistemas integrados de control ambiental, tales como: utilizar ordenadores domésticos, utilizar telecomandos, poder recibir señales enviadas en viajes, o utilizar sistemas controlados por voz.

\section{La experiencia japonesa}

Según estudios realizados en Japón, las necesidades específicas de los discapacitados y de las personas de edad avanzada son, en primer lugar, las de seguridad y las de ayuda junto a la de un sistema que provea información y permita el estudio y el esparcimiento en el hogar. En contra de lo esperado, la posibilidad de comprobar la salud en casa se consideró de menor importancia. Este control podría hacerse a través de un monitor conectado a la televisión que permite un chequeo, posibilitando una vida activa sin chequeos médicos frecuentes.

Según los investigadores japoneses, para una mayor generalización de las "casas inteligentes", las acciones a seguir son:

-Estandarizar las especificaciones relativas a los equipos y a los sistemas y a su expandibilidad.

-Explicar los servicios disponibles y mejorar la fiabilidad de los equipos.

-Proporcionar facilidades de financiación.

La compañía Sekisui House Incha ha desarrollado una casa prototipo destinada a discapacitados. Este prototipo está provisto de equipos automatizados específicamente diseñados para obtener las mayores prestaciones de cada función de la casa. Entre las instalaciones, hay una cocina, cuya mesa de trabajo, totalmente equipada, puede situarse a distintas alturas según las necesidades del usuario, mediante un dispositivo que se activa a través de un panel de pulsadores fácilmente accesible. El dormitorio está equipado con un dispositivo que permite variar la altura de la cama, hasta su desaparición en el techo, lo que proporciona un mayor espacio y mejora Tâ accesibilidad al eliminar obstáculos. Dispone también de un 
sistema de transporte desde el baño hasta el dormitorio en el que se puede situar un asiento, que está formado por una guía situada en el techo y que permite a los discapacitados moverse autónomamente.

El prototipo está provisto de un sistema electrónico de control, a través de cuya consola pueden gestionarse los distintos dispositivos de la casa o monitorizar los parámetros con el fin de regular las condiciones ambientales interiores además de explorar los externos. El sistema tiene paneles de control del teléfono, del vídeo-portero automático y de los sistemas de seguridad, alumbrado interior, climatización, bajada automática de persianas, difusión de audio, así como otras posibilidades a requerimiento del usuario. El sistema de control de la casa puede ser dotado posteriormente de un panel que incluya el plano de la casa, de modo que todos los dispositivos puedan ser fácilmente controlados en las distintas habitaciones.

\section{Investigación en la Unión Europea}

La UE ha impulsado iniciativas para abordar estos problemas desde distintos esquemas. El programa más directamente orientado es el denominado TIDE (Technology Initiative for Disabled and Elderly people). Empezó en 1991, con una fase piloto entre 1991 y 1994 en la que se financió el $50 \%$ del coste de los proyectos aprobados, con un montante total de $18 \mathrm{Mecus}$ aplicados en 21 proyectos, que abarcaban: tecnologías de control; incluyendo control del entorno, ayudas robotizadas, etc.; tecnologías de comunicación, incluyendo terminales, proceso de señales para sistemas de alarma, ayudas auditivas, etc.; tecnologías de sistemas integrados, incluyendo casas inteligentes y sistemas de navegación; técnicas de fabricación, incluyendo CAD/CAM para la fabricación de prótesis y ortosis. Estudios sobre normativa, ensayos, factores legales y económicos sobre el establecimiento de un mercado único para este tipo de productos.

La segunda fase, denominada puente, se desarrolló entre 1994 y 1997, incluyendo 55 proyectos con una dotación total de $42 \mathrm{Mecu}$, cubriendo aspectos de acceso a la tecnología y a servicios relacionados con ella, vida en casa y control remoto, movilidad y transporte, restablecimiento y mejora funcional y estudios sobre el mercado y los usuarios. En el programa de aplicaciones telemáticas (1994-98), que se refiere a aplicaciones de investigación y desarrollo en tecnologías de información y/o comunicación en áreas de interés social, se incluyó un sector sobre discapacitados y personas de edad avanzada como usuarios y sus necesidades en cuidados médicos, educación transporte etc., que puede considerarse como el heredero de TIDE, con un presupuesto de $70 \mathrm{Mecu}$. Las actividades incluidas se agrupan en dos líneas. La primera es el acceso a la tecnología y a los servicios incluyendo el acceso a la información, tecnologías de comunicación, sistemas integrados de apoyo a las actividades de vida independiente, educación trabajo y entretenimiento y sistemas de comunicación para aumentar la eficiencia y la eficacia de los servicios de apoyo a la vida independiente. La segunda línea se refiere a la compensación de deficiencias funcionales y mejora de las capacidades .

Otro programa de interés es el denominado RACE (Research in Advanced Communications in Europe). Se hace especial énfasis en los discapacitados y personas mayores ante los sistemas de comunicación de banda ancha del futuro, ante el mercado creciente que este sector representa. Se ha considerado la investigación en sistemas de videofono con el fin de monitorizar los servicios para la vida independiente de este grupo de personas, nuevos tipos de comunicación y desarrollo de nuevos terminales de entrada y salida, adaptados a los distintos tipos de discapacidad, se incluyen entre sus objetivos.

En el programa ACTS (Advanced Communication Technologies and Services) se incluyen las necesidades del grupo ante las comunicaciones móviles y se han ensayado nuevos servicios. En el programa ESPRIT (European Strategic Programme of Research in Information Technology), se ha hecho énfasis en las adaptaciones para la vivienda inteligente o el hogar del futuro, en los programas marco II y III, en tanto que en el IV se ha prestado especial atención al desarrollo de ayudas auditivas y al de nuevos miembros conectados al sistema nervioso.

Otros programas como HELIOS y HORIZON se orientan más hacia la integración social y laboral de los discapacitados, promoviendo redes de información, programas de integración laboral, vocacional y educativa, etc. En ellos participan unas 700 organizaciones nacionales.

La iniciativa COST (Cooperation Européenne dans le domaine de la recherche Scientifique et Technique) incluye también a países europeos fuera de la Unión. Su objeto es fomentar la cooperación, al financiar seminarios y reuniones técnicas en temas de interés común. Entre las acciones realizadas puede destacarse la 219, titulada "Future Telecommunications and Tele-Informatics Facilities for Disabled and Elderly People", iniciada en 1986. La acción A5 "Ageing and Technology" es más reciente, su objetivo es explorar las condiciones para una vida más independiente de las personas mayores, desde un punto de vista comparativo entre las distintas naciones europea y la influencia en ello de las nuevas tecnologías.

La iniciativa EUREKA es de cooperación tecnológica y tiene por objeto mejorar la productividad y la competitividad de las empresas. Los intervinientes definen sus objetivos y la financiación es a nivel nacional. La inclusión en EUREKA facilita la obtención de financiación lo que hace que sea grande el número de proyectos; en http://informesdelaconstruccion.revistas.csic.es 
1994 se pusieron en marcha 650 proyectos EUREKA y, entre sus múltiples finalidades, se encuentran temas de comunicaciones, nuevos materiales, robots y el entorno, automatización de la producción, tecnologías laser. Se han desarrollado aplicaciones sobre transportes para ancianos, lentes birrefringentes, instalaciones para control de energía doméstica, etc.

\section{Investigaciones futuras}

En aras a mejorar las condiciones de vida de los discapacitados y de las personas mayores es necesario realizar investigaciones, entre otros, en aspectos médicos, sociales, económicos y tecnológicos. Desde el punto de vista de la Domótica serán estos últimos en los que se hará mayor hincapié, pero siempre insertos en u orientados por los otros.

Las intervenciones pueden ser sobre el individuo o sobre el entorno. En el primer caso deben tenerse en cuenta las capacidades y discapacidades de los individuos y, en el segundo caso, los dispositivos y las barreras del entorno.

Respecto a las intervenciones sobre los individuos, debe investigarse sobre modelos de vida independiente, definirse grados de dependencia y métodos para abordarlos, lo que incluye el desarrollo de procesos de autosuficiencia, rehabilitación y de integración social. Soluciones para mantener a los más ancianos móviles, autónomos y autoatendidos; estrategias y métodos para mejorar las condiciones de vida independiente y modelos de vida integrada; el papel del entorno en el mantenimiento de vida independiente; adaptación de los vecindarios; entrenamiento de usuarios, etc.

Por lo que se refiere al entorno y sus cualidades de ayuda o de barrera, deben investigarse las intervenciones y las técnicas necesarias para mejorar las condiciones favorables y eliminar las barreras. Esto incluye el diseño para todos, el acceso a las tecnologías de información y de comunicaciones, el proyecto "ab initio" o la adaptación de los entornos domésticos, la elucidación de las necesidades de los usuarios en edificios de acuerdo con los tipos de discapacidad, la integración de tecnologías accesibles en los edificios, el conocimiento de los requerimientos de los usuarios, la evaluación de los edificios y entornos

Debe también investigarse sobre la interrelación entre la tecnología y la sociedad, el modo de cómo modela aquélla a la sociedad, el papel que juegan la estandarización, la legislación y los consensos sobre nivel de pruebas en el desarrollo o mejora de los productos, la eliminación de barreras lingüísticas en las documentaciones, el diseño de servicios, y la reorientacióm de las prácticas habituales para explotar las nuevas tecnologías.

Es necesario realizar un esfuerzo de investigación y de desarrollo en la aplicación de las tecnologías que se encuentran a disposición general, al caso particular de los discapacitados y de las personas mayores, pero ha de imprimirse un cierto giro a la orientación tradicional de proporcionar herramientas o servicios a los usuarios, para incluir también el proporcionar herramientas adecuadas a los prestadores de servicios. Es también importante reforzar el concepto de diseño para todos, haciendo que se tengan en cuenta las limitaciones impuestas por las discapacidades más habituales al diseñar los aparatos o servicios de uso general.

Es importante superar el localismo en el intento de dar soluciones tecnológicas, pues ello conduce al encarecimiento y falta de compatibilidad de los productos y a la duplicación de esfuerzos, más penosa cuanto más limitados son los recursos. Conviene, pues, asociarse a nivel multinacional y tratar de acceder a los fondos de apoyo que dedican organismos supranacionales, en particular la UE para el caso de los países que la formamos. Esta colaboración resulta enriquecedora para contemplar los problemas y las vías de solución en entornos y niveles de vida distintos, lo que conduce a soluciones con carácter más general y con una mayor viabilidad.

\section{BIBLIOGRAFIA}

(1) SATOSHI KOSEE: Development of design Guidelines of Dwellings for the Aging Society, Information technologies for buildings in a changing society, Capri, october 1992, Italian National Research Council. Central Institute for Building Technology.

(2) ROMOLO CONTINENZA, ASSUNTA D'INNOCENZO, KAZUHISA IKI: Ageing Services Management, Safety, Communication in Housing Schemes: Feasible and Desirable Developments, Information technologies for buildings in a changing society, Capri, october 1992, Italian National Research Council. Central Institute for Building Technology.

(3) ANNALISA MORINI: Intelligent House: A Comparison Between Italy and Japan, Information technologies for buildings in a changing society, Capri, october 1992, Italian National Research Council. Central Institute for Building Technology

(4) CONSTANTINE STEPHANIDIS, ARK KARSHMER: A Report on the Meeting and Workshop of the International Scientific Forum "Towards an Information Society for All", ACM Sigcaph, Newsletter, $n^{\circ} 62$, september 1998, pgs. 9-14, USA. 